\title{
Be prepared for prenatal diagnosis
}

\author{
'Genetic Disorders and the Fetus - Diagnosis, Prevention, And \\ Treatment', 7th edition \\ Edited by: Aubrey Milunsky and Jeff Milunsky \\ ISBN: 978-1-118-98152-8 \\ Published by: Wiley Blackwell: 2015 \\ Price: £200.00/€240.00 (Hardcover), £180.99/€216.99 (E-book)
}

\author{
European Journal of Human Genetics (2017) 25, 901; \\ doi:10.1038/ejhg.2017.67
}

As the previous editions, this is an incredibly useful volume. The editors have engaged multiple experts on specific topics, as well as provided the latest and most up-to-date information and discussions without leaving out broad topics, such as genetic counseling, medical/legal aspects, and ethical issues. Each chapter has useful figures and tables, including some lovely colored illustrations. Specific conditions are well covered, either by a chapter or under specific techniques. Non-invasive, prenatal screening using cellfree fetal DNA is well covered; however, the newer aspects of maternal physiology are not yet covered. The problem of fetal mosaicism is dealt with in many chapters, but not as an issue itself. A broad variety of fetal therapies, from infection to metabolic disease and structural abnormalities, are also covered as various topics and in two excellent chapters on medical therapy and fetal surgery.
Much has been learned about the molecular development of the placenta in the last decade. As the genetic pathways are luminated in the next decade, one can anticipate potential new therapies not yet imagined. Perhaps the most dramatic change since the last edition in 2010 is the wealth of molecular studies and the availability of whole genome sequencing for unusual situations.

The chapter on molecular genetics carefully outlines various considerations, the importance of reporting 'incidental' results, and how important epigenetics will be in the future. My only disappointment, because of a personal interest, is that fetal movement is not discussed. The importance of studying fetal movement for the diagnosis of arthrogryposis is not covered, nor is the observation of fetal behavior, which is a growing field of interest. Cross-referencing between chapters could also be helpful in the next edition.

In summary, this is a superb book, both a reference and a resource. It is clearly an essential book for anyone involved in prenatal diagnosis, and for those who want to learn more about it. It is hard to be critical of such a wonderful resource.

\section{CONFLICT OF INTEREST}

The author declares no conflict of interest.

Judith G Hall

Professor Emerita of Pediatrics and Medical Genetics, UBC \& Children's and Women's Health Centre of BC, Vancouver, BC, Canada

Dr Judith G Hall

E-mail: jhall@cw.bc.ca

\section{'Absolute clarity' and how to achieve it}

\author{
The Scientist's Guide to Writing-How to Write More Easily and \\ Effectively throughout Your Scientific Career \\ Written by: Stephen B Heard \\ ISBN: 9780691170220 (paperback)/9780691170213 (hardcover) \\ Published by Princeton University Press, 2016 \\ Price: Paperback— $\$ 21.95 / £ 17.95$ Hardcover— $\$ 59.95 / £ 49.95$
}

European Journal of Human Genetics (2017) 25, 901-902; doi:10.1038/ejhg.2017.58

This useful book is both a guide and a companion, with a key messagethat the scientist has to write with 'absolute clarity'. The author tells you how to do this, and he should know as he is a successful scientist and Editor, and sees papers and publications from both sides of the fence!
The book is arranged logically into seven sections. First up are some philosophical thoughts on the history of scientific writing from medieval scholar loaners to enlightenment scientific sharing, for example, Bacon's Salomon's House and why Newton bothered to publish at all!

The second section is on behaviour and the psychology of (scientific) writing. Most people have to work hard to become successful writers and it is a craft the scientist will have to learn. To bring his message home Heard quotes from the horror writer Stephen King, 'What is writing? The answer is telepathy.' You want your reader to understand your writing as easily as you do. A great emphasis in this book is to always think of the reader, and that you yourself can learn to be a better writer by your own critical reading of others. For the writing process itself there is much good advice on avoidance (for example, of getting started), distractions, feeling stuck (and how to conquer writers block), the peril of perfectionism, and what to do if 
you fear criticism and are reluctant to get others to review your work. Finally, there are wise words about 'early writing', and writing as you go through a project.

Section three is called 'Content and Structure', and concerns the mechanics of writing. Crucial is to 'find your story' (What's it all about?). As in a novel Heard suggests you set up an interesting and dramatic situation (by the Title and in the Introduction), tell the story (state your results), and then ensure that there is a resolution to the problem (the happy ending) in the Discussion. Heard talks of ways to ensure your story is clear and understandable, by use of wordstacks, concept maps and outlines. There follows sections on all parts of a paper (I particularly recommend his thoughts on Introduction). The advice is wonderful on the use of numbers (volume of numerical data), as is that on graphs, tables and figures-and how graphics should relate to text. Finally there is guidance on writing a Discussion, which Heard characterises and champions as 'turning data into knowledge'. Section three really should be compulsory reading for all scientific writers!

Section four details the nuts and bolts of writing scientifically, for example, the paragraph, which is 'a unit of logical organisation which is unified, coherent and distinct'. Thence follows chapters on essential matters, such as sentences, the passive or active voice, words themselves, and the wonderful Chapter 20 on Brevity, of which the first paragraph is 'Be brief'.

Revision of the manuscript is the subject of section five. There is much sage advice about the process of revision and reviews of a paper. This is divided into chapters on self-revision (a very necessary skill), friendly revision ('too often overlooked'), formal external review, and dealing with reviewers comments and editorial staff. Heard suggests that there are three types of reviewer comment: (1) where there is a problems - and a straightforward resolution; (2) where there seems to be a problem, but what it is and how to resolve it are unclear-you have not written clearly and your message has not been understood so, a significant rewrite is required; and (3) where you will likely be angry as the reviewer seems either not to have understood you or not to have read the paper properly-again a serious rewrite is needed, but be polite so that you don't add to the problem.

This book contains nearly everything you should know about scientific writing. Final sections deal with matters not covered elsewhere in the book, such as managing co-authorship and writing different types of articles. It ends with a chapter asking if scientific writing can be enjoyed, and can it include whimsy, humour and beauty? Read this book for the answers.

\section{CONFLICT OF INTEREST}

The author declares no conflict of interest.

Christopher Geoffrey Woods

The Clinical Medical School, University of Cambridge, Addenbrookes Hospital, Cambridge, UK E-mail:cw347@cam.ac.uk

\section{Thorough discussion of cancer research-thoughts against the main stream}

\author{
'Debating Cancer, The Paradox in Cancer Research', \\ Henry H Heng, Wayne State University, \\ School of Medicine, USA \\ ISBN: 9789814520843 \\ Published by: World Scientific: 2016 \\ Price: $£ 130.00 / £ 110.00$
}

European Journal of Human Genetics (2017) 25, 902-903; doi:10.1038/ejhg.2017.7

Cancer is, across the world, one among the five leading causes of death in humans. Thus, not only in USA but also elsewhere, cancer has been declared the 'biggest enemy of mankind', and during the past 30-50 years billions of private and governmental research money has been spent in the fight against cancer. While chromosomal aberrations and viral infections (oncogenes) were initially the research focus, for several decades the main attention has shifted to the 'gene mutation theory of cancer'. It is common sense that (a) there is a linear accumulation of mutations in a cell that stepwise evolves to a cancer cell; (b) mutations are different but specific for each cancer type; (c) leukemia behaves principally as solid tumors; and (d) once we accomplish a detailed study of each type of cancer, we will be able to find its Achilles heel and develop a drug against that type. A standard (and, notably, still almost only) example is that of chronic myelogenous leukemia (chronic phase), with $B C R-A B L$ gene fusion and Imatinib as a cure.

In his book Prof Heng acknowledges and summarizes all these historical developments of cancer research in a comprehensive and entertaining way. However, he also points out clearly that something went wrong in this research field at least during the last two decades. The book, surely mind-blowing for many readers, starts with the quotation of Dr Harold Varmus (former director of US National Cancer Institute), who wrote in his resignation letter concerning the current status of cancer research, perhaps including the 'gene mutation theory of cancer' and the yet widely unsuccessful ( $>5$ years) attempts to find common driver oncogenes by next-generation sequencing approaches: 'We've run out of money, it is time to start thinking'!

The book Debating Cancer, The Paradox in Cancer Research is an excellent example of how critical scientific thinking needs to be done, and the author invites the reader to and takes him on a journey of many unsolved questions and problems in this important field. In the first two chapters the present problem of the cancer research, being trapped by focusing only on the 'gene mutation theory of cancer', is outlined; Chapter 3 is about the existing alternative theories to explain cancer; and Chapter 4 reviews and discusses the somewhat disappointing practical results of the highly praised cancer genome sequencing project(s), and the inability to transfer the (often contradictory and/or 